\title{
Reducing therapeutic injection overuse through patients-prescribers Interaction Group Discussions in Kinondoni District, Dar es Salaam, Tanzania
}

\author{
AMOS Y. MASSELE ${ }^{1 *}$, YOHANA J. MASHALLA ${ }^{1}$, EDMUND J. KAYOMBO ${ }^{2}$, JULIUS D. \\ MWAISELAGE ${ }^{3}$, NATU E. MWAMBA ${ }^{4}$ and ISSESSANDA KANIKI ${ }^{5}$ \\ ${ }^{1}$ School of Medicine, University of Botswana, Gaborone, Botswana \\ 2Institute of Traditional Medicine, Muhimbili University of Health and Allied Sciences, Dar es Salaam, \\ Tanzania \\ ${ }^{3}$ Ocean Road Cancer Institute, Dar es Salaam, Tanzania \\ ${ }^{4}$ University of Dar es Salaam, Dar es Salaam, Tanzania \\ ${ }^{5}$ Kinondoni Municipal Council, Dar es Salaam, Tanzania
}

\begin{abstract}
Inappropriately prescription of injections has been reported in developing and developed countries. Previous studies in Tanzania showed that over $70 \%$ of patients attending out- patient clinics at private dispensaries received at least one injection per consultation, a value higher than WHO recommended target of $10 \%$. This is of concern considering the likelihood of adverse effects of possible use of unsafe syringes to transmit HIV, hepatitis B and C, poliomyelitis and added economic impact on the patient and the healthcare system. This study aimed to investigate the impact of Interaction Group Discussion on behavioural change on injection prescribing practices in ten selected public dispensaries in Kinondoni District, Dar es Salaam, Tanzania. Patient records of injection prescriptions were obtained covering the period three months prior to the study from 5 randomly selected control and 5 randomly selected intervention facilities. At each health facility IGDs were conducted for one month on mothers and prescribers followed by a survey 3 months after IGD to determine the impact of IGDs. Chi-square statistical calculations were made to compare data on the percent of prescriptions with an injection prescribed and in those conforming to national standard treatment guidelines (STG) between baseline and 3 months follow up. Results showed no significant difference between the percentage of prescriptions with an injection prescribed at baseline and 3 months follow-up in public dispensaries $\left(P>0.05, X^{2}\right.$ test). Prescribed injections that complied with STG was low at baseline and did not significantly improve 3 months after $\left(P>0.05, X^{2}\right.$ test). Comprehensive studies and sensitization of compliance to STG by prescribers are recommended.
\end{abstract}

Key words: Therapeutic injections, Interaction Group Discussion, prescribers, Tanzania

\section{Introduction}

The health care delivery system in Tanzania is at five levels including referral hospitals, regional hospitals, district hospitals, health centres and dispensaries. Health centres and dispensaries constitute the primary health care (PHC) facilities. The majority of the PHC facilities are headed by Clinical Officers and Assistant Clinical Officers. The Clinical Officers and Assistant Clinical Officers are middle level health professionals trained after successful completion of secondary education. Because of inadequate human resources to meet the demands of the PHC facilities, some facilities are managed by Maternal and Child Health Aids (MCHAs). With expanded student enrolment and output of the middle level health professionals from the training institutions the number of PHC facilities managed by MCHAs is rapidly decreasing.

\footnotetext{
${ }^{*}$ Correspondence: Prof. Yohana J.S. Mashalla; E-mail: Yohana.Mashalla@mopipi.ub.bw
} 
In 1980s, Tanzania liberalised provision of health care and the country saw an increasing number of privately owned clinics, dispensaries and hospitals. This was in addition to the many religious organisations which for years have contributed significantly to the provision of health care in the country (Mujinja 1995). The increased number of health facilities introduced a challenge to the health regulatory system in terms of monitoring and ensuring quality of the health services provided.

Inappropriate prescribing habits in many forms are commonly seen in developing countries including Tanzania (Greenhalgh, 1987; Wyatt 1993; Gumodoka et al. 1996; Massele \& Nsimba, 1999; Massele et al., 2001). Previous studies aimed at determining the extent of inappropriate prescribing habits conducted in Tanzania between 1999 and 2001 showed that in private dispensaries over $70 \%$ of patients attending out-patient clinics received at least one injection per encounter (Massele et al., 1993). This observation raises concern especially when considering the likelihood of adverse effects including transmission of HIV, hepatitis $\mathrm{B}$ and $\mathrm{C}$ resulting from injections using unsafe syringes. Injections are considered to be an effective route of administration of drugs into a patient. Injections are however generally more expensive than oral medications (Johana et al., 1996). Therefore inappropriate use of injections tends to add an economic cost on the patient and the health facility and healthcare system which already is facing financial constraints.

Many of the interventions introduced to address the problem of inappropriate prescription of injections by the Ministry of Health and Social Welfare in Tanzania, have been confined to educational training programmes in the form of seminars or workshops. Experiences have shown that such approaches have only little impact on behavioural change and prescribing habit. The Interactional Group Discussion has been reported to be an innovative approach to behavioural change (Johana et al., 1996). The purpose of this study was to test the impact of the Interactional Group Discussion on the use of injections in public primary health care facilities in Kinondoni District in the Dar es Salaam City in Tanzania. If such an intervention method proved to be successful, it could form an alternative to educational intervention to improve specific prescribing behaviors and overall quality of care.

\section{Materials and Methods}

\section{Study participants and data collection}

The study was carried out from October 2005-June 2006. The aim of the intervention was to assess the efficacy of an innovative behavioural intervention, namely Interaction Group Discussion (IGD), for reducing overuse of injections by health care workers and patients attending at PHC facilities in Kinondoni, Dar es Salaam, Tanzania. Baseline survey was carried out from October 2005 to February2006. The intervention was carried out in March 2006 and post intervention survey was carried out in June 2006. Prescribers including Clinical officers, and other paramedics from selected intervention dispensaries and an equal number of patients in the community (mostly mothers and a small number of male participants) were involved in the study. The participants were paired in the IGD with prescribers from a different dispensary. Each group consisted of 6 prescribers and 6 patients. IGD was conducted once for each group lasting between 90-120 minutes.

The themes of the IGDs included an exploration of the perceptions of the participants about being included in such a group, presentation and discussion of discrepancies between prescriber's and patient's beliefs and motivations regarding 
injection use. The exploration of feelings was important because the groups were heterogeneous, and each participant had his or her own role in the community. The IGD further explored motives among participants for the use of injections.

The moderators for each IGD included a social scientist and a clinical pharmacologist who together facilitated interactions among participants in the IGD. The social scientist focused more on the subtle confrontation regarding the discrepancies between prescribers' and patients' beliefs, while the clinical pharmacologist presented scientific information regarding proper use of injections. Participants were also guided to discuss on the proper principles of injection use and the possible risks of their misuse. It is envisioned that establishment of peer norms about correct injection behaviour in the groups would also help to bring about cooperation and consensus on the proper use of injections.

\section{Evaluation of the impact of IGDs on injection use}

Retrospective case records obtained from dispensary out-patient registers were used to establish baseline data on the proportion of patients receiving injection and injections conforming to the national Standard Treatment Guideline (STG) 3 months before the intervention. For comparison of the impact of the IGD a similar survey was carried out 3 months after the intervention was completed. WHO Guidelines (WHO, 1993) were used to randomly selected 30 case records per month. Total of 900 case records were obtained from the 10 public dispensaries 3 months before IGD and the same number of case records was obtained 3 months after IGD. Data was collected by research assistants trained in the methods recommended by WHO for collecting this type of data in health facilities. Data collectors were blinded to the study conditions of individual dispensaries and collection of data was supervised and the accuracy validated by members of the study team.

\section{Data analysis}

The collected data was analyzed by SPSSVersion11) and significant level determined by Chi-square test.

\section{Ethical clearance}

Both oral and written consent was obtained from consumers and prescribers before the IGD. All participants were free to withdraw from the study any time if they so wished without any penalty. The study received approval of the Kinondoni Municipal Hospital Research Ethics Committee. Information obtained from the IGD was recorded manually by note taking and tape recorder. The qualitative data was transcribed by the social scientist.

\section{Results}

There was active interaction among participants during the discussions. In the IGD group, the participants suggested that most patients do not demand for an injection. This observation was in contrast to the prescribers who argued that the use of injections was based on patients' demand.

The average percentage of prescriptions with an injection prescribed 3 months before and after IGD is presented in Table 1. The percentage of prescriptions where an injection had been described was not statistically significant $(P>0.05)$ between the baseline and three months post IGD data in both control and intervention facilities. The percentages 
of injection prescriptions conforming to the Standard Treatment Guidelines (STG) in Tanzania are presented in Table 2. The percentage of prescriptions conforming to STG was not statistically significant $(P>0.05)$ between baseline and three post IGD in the intervention and control groups.

Table 1: Percent of prescriptions with an injection prescribed in the control and intervention arm before and after IGD

\begin{tabular}{|c|c|c|c|c|}
\hline $\begin{array}{l}\text { Randomized } \\
\text { group }\end{array}$ & $\begin{array}{l}\text { No. of case } \\
\text { records }\end{array}$ & $\begin{array}{l}\text { Average \% of } \\
\text { prescriptions } 3 \\
\text { months before }\end{array}$ & $\begin{array}{l}\text { Average \% } \\
\text { prescriptions } \\
\text { months after }\end{array}$ & P-value \\
\hline Control & 900 & $17.9^{*}$ & $13.6^{*}$ & $>0.05^{*}$ \\
\hline Intervention & 900 & $24.2+$ & $26.7+$ & $>0.05+$ \\
\hline
\end{tabular}

$\mathrm{X}^{2}$ test ${ }^{*}=\mathrm{P}>0.05$ (not statistically significant difference between baseline and 3 months post IGD in control and $+=\mathrm{P}>0.05$ baseline and 3 months post IGD in the intervention arms

Table 2: Percent of prescriptions conforming to STG in the control and intervention arm before and after IGD

\begin{tabular}{llllll}
\hline $\begin{array}{l}\text { Randomized } \\
\text { group }\end{array}$ & $\begin{array}{l}\text { No. of case } \\
\text { records }\end{array}$ & $\begin{array}{l}\text { Average \% of } \\
\text { prescriptions } 3 \\
\text { months before }\end{array}$ & $\begin{array}{l}\text { Average \% of } \\
\text { prescriptions } \\
\text { months after }\end{array}$ & $\begin{array}{l}\text { P-value } \\
\text { monther }\end{array}$ \\
\hline Control & 900 & $36^{*}$ & $44^{*}$ & $>0.05^{*}$ \\
Intervention & 900 & $29 \infty$ & $27 \infty$ & $>0.05 \infty$ \\
\hline
\end{tabular}

$\mathrm{X}^{2}$ test ${ }^{*}=\mathrm{P}>0.05$ (not statistically significant difference between baseline and 3 months post IGD in control and $\infty \mathrm{P}>0.05$ baseline and 3 months post IGD in the intervention arms

\section{Discussion}

This study was an attempt to validate IGD as an alternative method to the conventional educational interventions used to improve specific prescribing behaviours and overall quality of care in the health sector in Tanzania. From the discussions in this study it is clear that participants were free to talk and express their views about injection prescribing habits, that prescription of injections was not dictated by demand of injections by the patients. This finding suggests that there is some level of community concern on over prescription of injections by prescribers in the studied primary health centre facilities. This is an interesting observation which contradicts the argument by prescribers that prescription of injections is influenced by patients demanding for injections. The factors influencing injection prescriptions were not explored in this study, therefore, the challenge remains to be further explored.

The literature (Johana et al., 1996; Goel et al., 1997) suggests that IGDs have a positive impact in reducing injection overuse. It has also been reported in South East Asia that a "face to face educational" intervention carried out for counter attendants resulted in significant short-term improvements for anti-diarrhoeal drugs and instructions given to customers on diarrhoea treatment (Ross-Degnan et al., 1996). Our findings do not support these observations. Prescription of injections did not change within the period of the study. Our study lasted for three months only a period which may not have been long enough to bring a significant behavioural change. The findings however, seem to support other studies which have demonstrated that changing behaviours or individuals performance is a difficult task that requires constant sensitisation/training, monitoring and supervision for 
a reasonable length of time (Goel et al., 1997; Tarimo et al., 2001; Berwick, 2003), otherwise people tend to forget and usually return back to their original (baseline) performance practices they are used to (WHO 1993; Bloland \& Ettling, 1999; Fevre \& Barnish, 1999). Therefore, for an intervention to be sustained it must be given regularly.

Thus, over prescription of injections seem to still be a problem among prescribers whose extent still has to be explored. This study was carried out at selected primary health facilities in Kinondoni and comparable studies in other health centres, district and regional hospitals in the country are required. It is recommended that in order to determine the extent of over prescription of injections in Tanzania a large scale studies should be designed and carried out at all levels of the health care system in the country. Heads of health facilities should be made aware of the existence of such practices and should be encouraged to carry out situational analysis at their facilities and develop strategic interventions for reducing over prescription of injections. The Ministry of Health and Social Welfare should ensure that STG are accessible to all health facilities and where opportunities arise use professional associations annual conferences and other platforms to raise awareness of prescribers and the community on the Guidelines, and train prescribers on the use of the guidelines for ensuring compliance and improved prescribing habits.

\section{Acknowledgements}

The work was supported by the Rockefeller Foundation Grant to the University of Dar es Salaam to support the project on Enhancing Critical Thinking on Poverty Reduction in Tanzania.

\section{References}

Berwick, D.M. (2003) Disseminating innovations in health care. Journal of American Medical Association 289, 196-197.

Bloland, P.B. \& Ettling, M. (1999) Making malaria-treatment policy in the face of drug resistance. Annals of Tropical Medicine \& Parasitology 93, 5-23.

Fevre, E.M. \& Barnish, G. (1999) Malaria treatment policies: when and how should they be changed? Annals of Tropical Medicine E Parasitology 93, 549-560.

Goel, P.K., Ross-Degnan, D., McLaughlin, T.J. \& Soumerai, S.B. (1997) Retail pharmacies in developing, countries: a behaviour and intervention framework. Social Science and Medicine 42, 1151-1161.

Greenhalgh, T. (1987) Drug prescription and self-medication in India: an exploratory survey. Social Science and Medicine 25, 307-318.

Gumodoka, B., Vos, J., Berege, Z.A., van Asten, H.A., Dolmans, W.M. \& Borgdorff, M.W. (1996) Injection practices in Mwanza Region, Tanzania: prescriptions, patient demand and sterility. Tropical Medicine and International Health 1, 874-880.

Johana, E., Prawitasari, H., Sri Suryawati, Sulanto S, Danu, S. \& Budiono, S. (1996) Interactional group discussion: Results of a controlled trial using a behavioural intervention to reduce the use of injections in public health facilities. Social Science and Medicine 42, 1177-1183.

Massele, A.Y. \& Nsimba, S.E.D. (1999) Comparison of drug utilisation in public and private Primary Health Care Clinics in Tanzania. East African Medical Journal 74, 420-422. 
Massele, A.Y., Nsimba, S.E.D. \& Rimoy, G. (2001) Prescribing habits in Church owned Primary Health Care facilities in Dar es Salaam and other Tanzanian Coast Regions. East African Medical Journal 78, 510-514.

Massele, A.Y., Ofori-Adjei, D. \& Laing, R.O. (1993) Laing. A study of prescribing patterns with special reference to drug use indicators in Dar es Salaam region, Tanzania. Tropical Doctor 23, $104-107$.

Mujinja, P.G.M. ( 1995) A National Study: The relationship between the government and mission hospitals in Tanzania. A report of the Ministry of Health United Republic of Tanzania.

Ross-Degnan, D., Soumerai, S., Goe1, P., Bates, J., Makhu, J., Dondi, N., Sutoto, D., Adi, Ferraz-Tabor, L. \& Hogan, R. (1996) The impact of face to face educational outreach on diarrhoea treatment in pharmacies. Health Policy Planning 11, 308-318.

Tarimo, D.S., Minjas, J. \& Bygberg, IC. (2001) Perceptions of chloroquine efficacy and alternative treatments for uncomplicated malaria in children in a holoendemic area of Tanzania: implications for the change in policy. Tropical Medicine $\mathcal{E}$ International Health 6, 992-997.

Wyatt, H.V. \& Mahadevan, S. (1993) Unnecessary injections and poliomyelitis. Indian Journal of Paediatrics 60, 327-329.

WHO (1993) How to investigate drug use in health facilities. WHO/DAP/93.1 Manual. World Health Organization, Geneva. 